\title{
Toxicity Syndromes, Patient-Related Clinical Indicator of Toxicity Burden Induced by Intensive Triplet Chemotherapy-Based Regimens in Gastrointestinal Cancers With Metastatic Disease
}

\section{OPEN ACCESS}

Edited by:

Nicola Silvestris,

University of Bari Aldo Moro, Italy

Reviewed by:

Haruhiko Sugimura,

Hamamatsu University School of Medicine, Japan

Oronzo Brunetti,

John Paul II Cancer Institute (IRCCS), Italy

*Correspondence:

Enrico Ricevuto

enrico.ricevuto@univaq.it

Specialty section

This article was submitted to

Gastrointestinal Cancers,

a section of the journal

Frontiers in Oncology

Received: 19 September 2019 Accepted: 31 January 2020

Published: 20 February 2020

Citation:

Bruera G and Ricevuto E (2020) Toxicity Syndromes, Patient-Related Clinical Indicator of Toxicity Burden Induced by Intensive Triplet Chemotherapy-Based Regimens in

Gastrointestinal Cancers With Metastatic Disease.

Front. Oncol. 10:172.

doi: 10.3389/fonc.2020.00172

\author{
Gemma Bruera $^{1,2}$ and Enrico Ricevuto ${ }^{1,2 *}$ on behalf of Oncology Network ASL1 \\ Abruzzo Italy ${ }^{1}$ \\ 1 Oncology Territorial Care, S. Salvatore Hospital, Oncology Network ASL1 Abruzzo, University of L'Aquila, L'Aquila, Italy, \\ ${ }^{2}$ Department of Biotechnological and Applied Clinical Sciences, University of L'Aquila, L'Aquila, Italy
}

Background: Cancer treatments induce symptoms/signs superimposing on individual patient's clinical status, determining heterogenous toxicity syndromes (TS). We reviewed intensive first line triplet chemotherapy-based regimens in metastatic gastro-intestinal cancers (mGl), based on Flr/FOx schedule, fluorouracil and weekly alternating irinotecan/oxaliplatin, to point out limiting TS (LTS) relevance.

Methods: Metastatic colo-rectal (mCRC), pancreatic ductal adenocarcinoma (mPDAC), gastric carcinoma (mGC) patients were enrolled by careful decision-making including age, performance status (PS), and comorbidity status in real life phase II studies: Flr-B/FOx adding bevacizumab (B) overall, Flr-C/FOx-C adding cetuximab (C) in KRAS/NRAS wild-type mCRC; FIr/FOx in mPDAC; FD/FOx adding docetaxel (D) in mGC. Toxicity, individual LTS, LT alone (LTS-single site, LTS-SS) or associated to other limiting/G2 toxicities (LTS-multiple sites, LTS-ms) were evaluated, compared by chi-square test. In Flr-C/FOx-C, 5-fluorouracil/irinotecan pharmacogenomic biomarkers, 5-fluorouracil degradation rate (5-FUDR), SNPS ABCB1, CYP3A4, DYPD, UGT1A1 were evaluated, related with LTS.

Results: Flr-B/FOx, Flr-C/FOx-C in mCRC, Flr/FOx in mPDAC, FD/FOx in mGC, showed activity, efficacy, toxicities similar to reported triplet regimens. LTS: mCRC Flr-B/FOx 44\%, LTS-ms 24\%, LTS-ss 20\%, in young-elderly 46\%, LTS-ms significantly increased vs. LTS-sS; Flr-C/FOx-C 65.5\%, significantly increased LTS-ms vs. LTS-sS, in youngelderly 83\%; mPDAC Flr/FOx 27.5\%, mostly LTS-ms, in young-elderly 38.4\% all LTS-ms; mGC FD/FOx 30\%, all LTS-ms, in young-elderly 25\%. Reduced FUDR, SNPs CYP3A4, UGT1A1, >1 positive pharmacogenomic biomarkers were prevalent in patients with gastrointestinal LTS.

Conclusions: LTS is an innovative clinical parameter of toxicity burden, differential treatment-related TS in individual patient. LTS can evaluate pharmacogenomic biomarkers predictive relevance to select $\mathrm{mGl}$ patients fit for intensive treatments, at risk of limiting gastrointestinal toxicity. 
Trial Registrations: The trials were registered at Osservatorio Nazionale sulla Sperimentazione Clinica dei Medicinali (OsSC) Agenzia Italiana del Farmaco (AIFA) Numero EudraCT 2007-004946-34, and Osservatorio Nazionale sulla Sperimentazione Clinica dei Medicinali (OsSC) Agenzia Italiana del Farmaco (AIFA) Numero EudraCT 2009- 016793-32.

Keywords: individual limiting toxicity syndromes, intensive first line, metastatic gastrointestinal cancer, real life, timed-flat-infusion 5-fluorouracil administration, triplet chemotherapy-based regimens, weekly alternating schedule

\section{INTRODUCTION}

\section{The Need of Patient-Related Clinical Indicator of Toxicity Burden}

Addition of more drugs in a chemotherapy combination requires the design of proper schedule and doses, to provide the balance between projected/received $(>80 \%)$ dose intensity (DI) and treatment-related toxicity (1). This clinical balance is even more challenging to realize for intensive triplet chemotherapybased regimens that demonstrated to increase clinical outcome in fit metastatic gastro-intestinal (mGI) cancer patients (118). Clinical status of the individual patient, also depending from metastatic tumor extension, is the most important variable justifying differential toxicity in individual patients. Thus, patients treated with triplet chemotherapy-based regimens should be enrolled by careful decision-making including age, performance status (PS), and comorbidity status (19).

The conventional evaluation of toxicity of cancer treatments depends upon grading of the clinical relevance of each toxicity symptom and sign, by evaluation of the type, prevalence and intensity of toxicity, more specifically of limiting (G3-4) toxicity, related to the administered treatment regimen, and determined by National Cancer Institute Common Toxicity Criteria (NCICTC, version 4.0). Thus, safety profile and toxicities induced by cancer treatments are conventionally defined, according to the number of administered cycles and treated patients, as severe (G3-4), moderate (G2), mild (G1), or absent. This evaluation does not individually describe the toxicity burden experienced in a single patient, defining a spectrum of toxicity syndromes (TS). To better evaluate individual toxicity of intensive treatments, we introduced the concept of Limiting Toxicity Syndromes (LTS), consisting of at least a limiting toxicity alone (LTS-single site, LTS-ss) or associated to other limiting or G2-3 non-limiting toxicities (LTS-multiple sites, LTS-ms) (2, 4, 11, 14, 20). More,

\footnotetext{
Abbreviations: B, bevacizumab; C, cetuximab; CIRS, Cumulative Illness Rating Scale; CPT-11, irinotecan; D, docetaxel; DI, dose-intensity; IADL, Instrumental Activity of Daily Living; LTS, limiting toxicities syndromes; LTS-ms, limiting toxicity syndrome multiple sites; LTS-ss, limiting toxicity syndrome single site; mCRC, metastatic colorectal cancer; mGC, metastatic gastric cancer; mGI, metastatic gastro-intestinal; mPDAC, metastatic pancreatic ductal adenocarcinoma; NCI-CTC, National Cancer Institute-Common Toxicity Criteria; ORR, objective response rate; OS, overall survival; OXP, oxaliplatin; PFS, progression-free survival; PS, performance status; rDI, received dose-intensity; SNP, Single Nucleotide Polimorphisms; TFI, timed-flat-infusion; TS, toxicities syndromes; yE, young-elderly; 5-FU, 5-fluorouracil; 5-FUDR, 5-fluorouracil degradation rate.
}

symptoms and signs induced by cancer medical treatments add up to clinical general and cancer-related status of the individual cancer patient determining heterogenous TS. A clinical parameter of patient-related toxicity, integrated with conventional treatment-related toxicity evaluation, represents an unmet need in clinical oncology, to verify the occurrence of the spectrum of different toxicities in individual patients, and in a cohort of patients at differential intensity.

We proposed the analysis of TS in different studies developing intensive triplet chemotherapy-based regimens in gastro-intestinal cancer patients with metastatic disease $(2,4$, $11,14,20)$, to evaluate the clinical relevance of the integration of patient-related to conventional treatment-related toxicity, to more properly weigh toxicity with treatment-related activity and clinical outcome, and contributing to better address selection of patients suitable for intensive medical treatments. To this aim, LTS represent an innovative clinical parameter of patient-related toxicity burden, indicating global and individual toxicity, consisting of a differential spectrum and intensities of TS, related to administered treatment, according to clinical patient (age, performance status, comorbidity status), and metastatic extension.

\section{The Model: Intensive First Line Triplet Chemotherapy-Based Regimens in Metastatic Gastrointestinal Cancers}

We previously developed doublet chemotherapy schedule of $12 \mathrm{~h}$ (10 p.m. to 10 a.m.) timed-flat infusion (TFI) 5-FU (21), associated to irinotecan (CPT-11), safely administered at high 5-FU/DI without leucovorin addiction, with a good tolerability profile, and with activity and efficacy equivalent to reported doublet regimens in metastatic colorectal cancer (mCRC). Then, we designed FIr/FOx triplet chemotherapy schedule by splitting weekly TFI/5-FU (22), and weekly alternating CPT11 and oxaliplatin (OXP) at DIs 1800, 80, and $40 \mathrm{mg} / \mathrm{m}^{2} / \mathrm{w}$, respectively, and reported objective response rate (ORR) $66.7 \%$, progression-free survival (PFS) 12 months, and overall survival (OS) 20 months as first line mCRC treatment. Thus, we developed intensive first line regimens, based on FIr/FOx triplet chemotherapy schedule: FIr-B/FOx (2) and FIr-C/FOx-C (4), respectively adding bevacizumab (B) or cetuximab (C), in mCRC; FIr/FOx in metastatic pancreatic ductal adenocarcinoma (mPDAC) (11); FD/FOx in metastatic gastric cancer (mGC), including docetaxel (D) in the same schedule (14). 


\section{MATERIALS AND METHODS}

Patients treated with triplet chemotherapy-based regimens were enrolled by decision-making process including age, performance status (PS), and comorbidity status evaluated by Cumulative Illness Rating Scale (CIRS) (19). CIRS stage was defined as: primary, absent or mild grade comorbidities, and independent Instrumental Activity of Daily Living (IADL); intermediate, $<3$ mild or moderate grade comorbidities, and dependent or independent IADL; secondary, more than three comorbidities or a severe comorbidity, with or without dependent IADL. Patients with primary or intermediate CIRS stage were enrolled in intensive first line treatment regimens.

Proposed regimens were approved by Agenzia Italiana del Farmaco for administration in label for treatment in Italian public hospitals, and published in Gazzetta Ufficiale Repubblica Italiana ("Elenco dei Medicinali erogabili a totale carico del Servizio Sanitario Nazionale," Gazzetta Ufficiale Repubblica Italiana N.1, 2 Gennaio 2009). MCRC clinical trials were approved by Local Ethical Committee (Comitato Etico, Azienda Sanitaria Locale n.4 L'Aquila, Regione Abruzzo, Italia), and conducted in accordance with Declaration of Helsinki. PDAC study was approved by the Regional Review Board (Regione Abruzzo, Italia, according to D.G.R. n.489, 25/05/2007). All patients provided written, informed consent concerning the proposed treatment, and biological evaluations.

Figure 1 shows recommended triplet chemotherapy-based regimens developed as first line treatment in mGI cancers. In mCRC, FIr-B/FOx schedule (2): weekly TFI/5-FU $900 \mathrm{mg} / \mathrm{m}^{2}$, days 1-2, 8-9, 15-16, 22-23; CPT-11 $160 \mathrm{mg} / \mathrm{m}^{2}$ days 1,15; OXP $80 \mathrm{mg} / \mathrm{m}^{2}$, days 8,22 ; every 4 weeks; B $5 \mathrm{mg} / \mathrm{kg}$, days 1,15 . In KRAS/NRAS wild-type mCRC, FIr-C/FOx-C (Figure 1B) (4) weekly C loading dose $400 \mathrm{mg} / \mathrm{m}^{2}$, followed by $250 \mathrm{mg} / \mathrm{m}^{2}$, was added to triplet FIr/FOx schedule; in subsequent dosefinding steps, 5-FU and CPT-11 were recommended at doses 750 and $120 \mathrm{mg} / \mathrm{m}^{2}$, respectively. In mPDAC, FIr/FOx schedule (Figure 1C) (11): TFI $/ 5-F U ~ 900 ~ m g / \mathrm{m}^{2} /$ die weekly; CPT-11 160 $\mathrm{mg} / \mathrm{m}^{2}$, days 1 and 15; OXP $80 \mathrm{mg} / \mathrm{m}^{2}$, days 8 and 22. Drug's doses were modulated in patients reporting PS 2 , and/or $\geq 75$ years, secondary CIRS stage, and/or liver laboratory tests upper normal limit ( $\geq \mathrm{G} 2$ hypertransaminasemy at baseline). In $\mathrm{mGC}$, FD/FOx (Figure 1D) (14): TFI/5-FU $1000 \mathrm{mg} / \mathrm{m}^{2} /$ die weekly; D $50 \mathrm{mg} / \mathrm{m}^{2}$ days 1,15 ; OXP $80 \mathrm{mg} / \mathrm{m}^{2}$ days 8,22 ; every 4 weeks.

Conventional toxicity analysis was registered by National Cancer Institute Common Toxicity Criteria (NCI-CTC, version 4.0). To better evaluate toxicity of intensive treatments in the individual patient, LTS, consisting of at least a limiting toxicity alone (LTS-ss) or associated to other limiting or G2-3 nonlimiting toxicities (LTS-ms) were evaluated $(2,4,11,14,20)$. The rates of LTS-ms and LTS-ss were compared by Chi-square test (23). More, in FIr-C/FOx-C study, exploratory analysis of 5-FU/CPT-11 pharmacogenomic biomarkers, specifically 5-FU degradation rate (5-FUDR), defining reduced metabolizers if $<1.2 \mathrm{ng} / \mathrm{mL} / 10^{6}$ cells $/ \mathrm{min}$, and Single Nucleotide Polimorphisms (SNPs) ABCB1 (C3435T, C1236T), CYP3A4 (1B, 53), DYPD1 (IVS14+1, A166G), UGT1A1 (28) were preliminarily related with the occurrence of $\operatorname{LTS}(4,24,25)$.

\section{RESULTS}

\section{Clinical Outcome and Toxicity}

In mCRC patients, FIr-B/FOx and FIr-C/FOx-C were recommended at different 5-FU/CPT-11 doses, 900/160 and $750 / 120 \mathrm{mg} / \mathrm{m}^{2}$, respectively (Table 1) $(2,4)$. In overall and KRAS/NRAS wild-type mCRC patients treated with FIr-B/FOx, and FIr-C/FOx-C, respectively, median received DI (rDI) per cycle for all the drugs at the recommended doses were $\geq 80 \%$ $(2,4)$. Reported ORR were, respectively, 82 and $78 \%$, PFS equivalently 12 months, OS 28 and 23 months. In young-elderly (yE) mCRC patients ( $\geq 65<75$ years) treated with FIr-B/FOx, ORR was 79\%, median PFS 11 months, median OS 21 months (20). In yE mCRC patients treated with FIr-C/FOx-C, median rDIs per cycle were $<80 \%$ : 5 -FU $71 \%\left(1066 \mathrm{mg} / \mathrm{m}^{2} / \mathrm{w}\right)$; CPT- 11 $76 \%\left(45.5 \mathrm{mg} / \mathrm{m}^{2} / \mathrm{w}\right) ;$ OXP $66 \%\left(26.5 \mathrm{mg} / \mathrm{m}^{2} / \mathrm{w}\right)$; C $71 \%(178.5$ $\left.\mathrm{mg} / \mathrm{m}^{2} / \mathrm{w}\right) ; \mathrm{yE}$ patients showed significantly worse OS compared to non-elderly $(P$ 0.045) $(4)$. Overall, FIr-C/FOx-C treatment was discontinued due to LT in $48 \%$ patients, prevalently due to diarrhea. Prevalent G3-4 toxicities by patients treated with FIr-B/FOx and FIr-C/FOx-C at recommended doses were, respectively: diarrhea 28 and 23\%, asthenia 6 and 15\%, neutropenia $10 \%$ and none, vomiting 4 and $8 \%$, mucositis $6 \%$ and none, hypertension $2 \%$ and none, hypokalemia $2 \%$ and none, hypertransaminasemy 4 and $8 \%(2,4)$.

In mPDAC patients, FIr/FOx showed ORR 53\%, PFS 4 months, OS 11 months; among yE/old-elderly patients, median PFS 4 months, and median OS 5 months (11). Elderly patients did not showed significantly worse PFS and OS compared to nonelderly patients. Patients with PS 2 showed significantly worse OS compared with PS $0-1$ patients $(P=0.022)$. In mPDAC patients treated with FIr/FOx, median rDI per cycle were: 5-FU 70.4\% (1268.5 mg/m²/w); CPT-11 70\% $\left(56 \mathrm{mg} / \mathrm{m}^{2} / \mathrm{w}\right)$; OXP $72.5 \%\left(29 \mathrm{mg} / \mathrm{m}^{2} / \mathrm{w}\right)$. In yE, 5 -FU $83.3 \%$, CPT-11 80\%, OXP $85 \%$. Overall, $17 \%$ discontinued FIr/FOx treatment due to LT. Limiting cumulative G3-5 toxicities were: diarrhea $17 \%$, asthenia $14 \%$, neutropenia $17 \%$, mucositis $6 \%$, hypokaliemia $7 \%$, hypertransaminasemia 7\%, nausea/vomiting, hypoalbuminemia, anemia, thrombocytopenia $3 \%$, respectively. One case of toxic death $(3 \%)$ was observed.

In mGC patients, FD/FOx at recommended 5-FU 1000 $\mathrm{mg} / \mathrm{m}^{2} /$ day, D $50 \mathrm{mg} / \mathrm{m}^{2}$ and OXP $80 \mathrm{mg} / \mathrm{m}^{2}$, showed ORR $60 \%$, PFS 6 months, OS 17 months (14). Median rDI were: 5-FU 82.9\% (1657.5 mg/m²/week); D 91\% (22.75 mg/ $/ \mathrm{m}^{2} /$ week); OXP $83.8 \%$ $\left(33.5 \mathrm{mg} / \mathrm{m}^{2} /\right.$ week). Cumulative G3-4 toxicities were represented by: asthenia $20 \%$, neutropenia $50 \%$, leucopenia $20 \%$, mucositis and hypoalbuminemia $10 \%$.

\section{Evaluation of Individual Toxicity Syndromes (TS) and Relationship With Companion Pharmacogenomic Analysis}

In KRAS/NRAS wild-type mCRC patients treated with FIrB/FOx, overall LTS were observed in 22 patients (44\%) (Table 2): LTS-ms 12 (24\%); LTS-ss 10 (20\%) (2). LTS-ms characterized by $\geq 2$ LT 2 (4\%); LT associated to other, at least G2, non-limiting toxicities 10 (20\%). In yE mCRC treated with FIr-B/FOx, LTS were observed in 13 out of 28 patients (46\%): LTS-ms, 11 (39\%); 

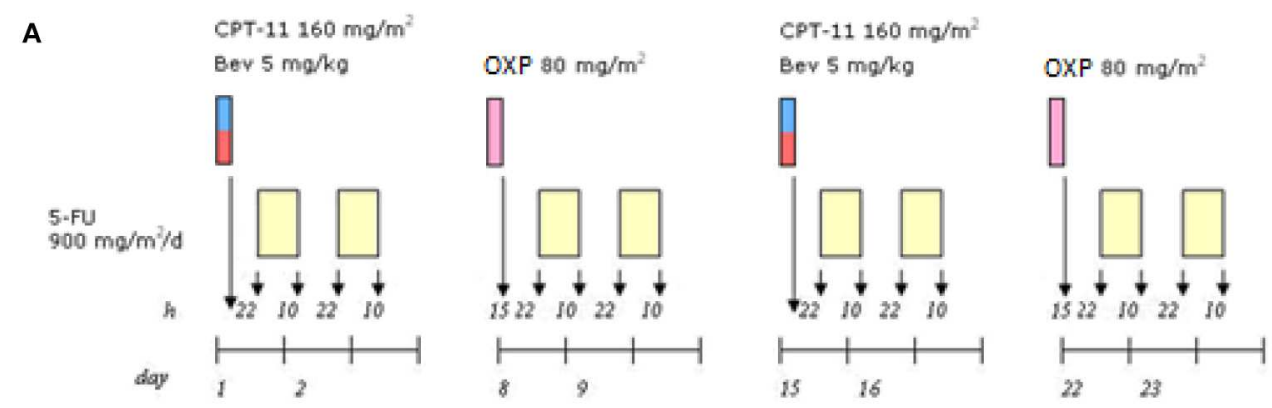

FIr-B/FOx schedule
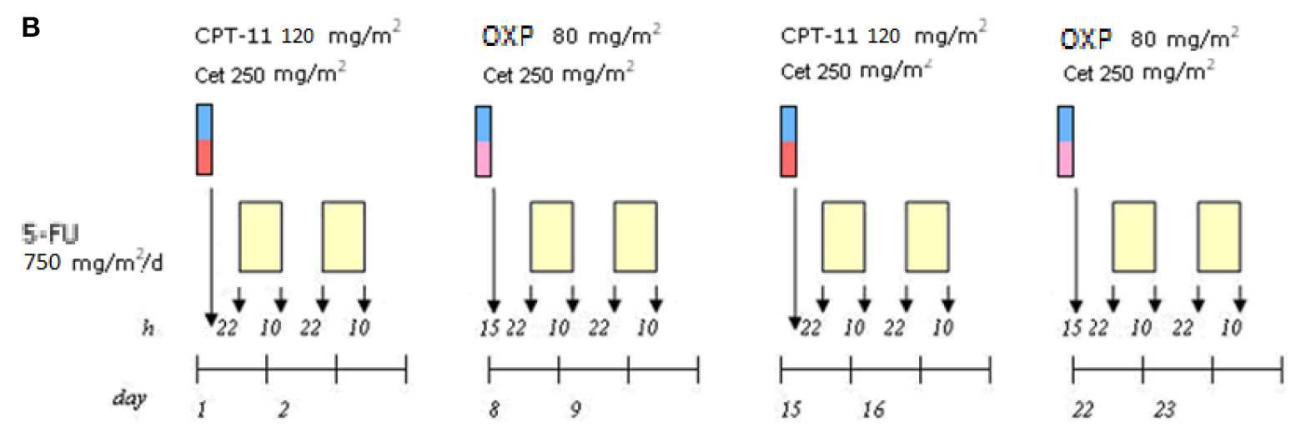

FIr-C/FOx-C schedule
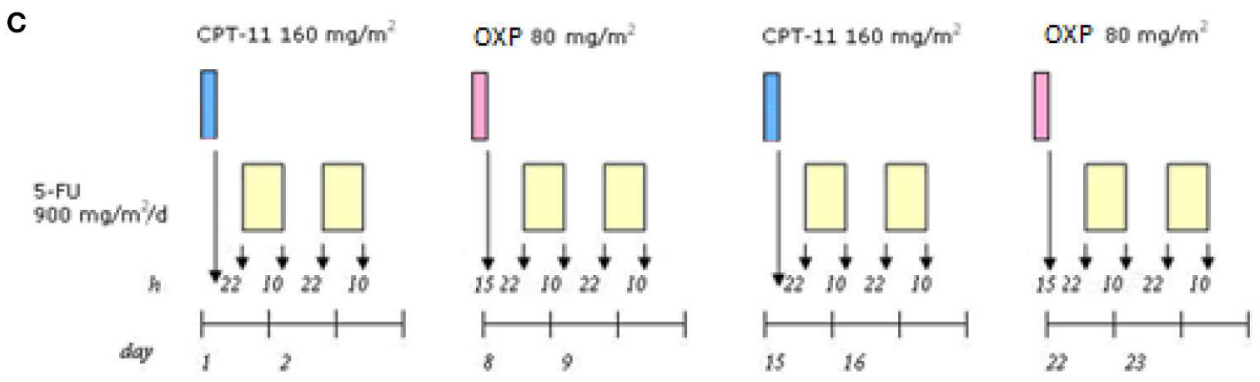

\section{FIr/FOx schedule}
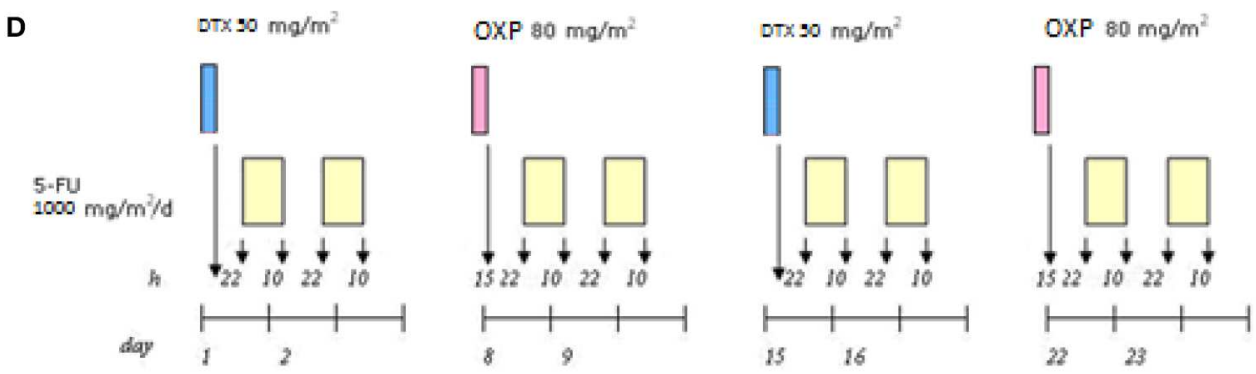

\section{FD/FOx schedule}

FIGURE 1 | Triplet chemotherapy-based schedules. (A) Flr-B/FOx schedule in metastatic colorectal cancer. (B) Flr-C/FOx-C schedule in metastatic colorectal cancer. (C) Flr/FOx in metastatic pancreatic ductal adenocarcinoma. (D) FD/FOx in metastatic gastric cancer. 
TABLE 1 | Treatment regimens, clinical outcome, and safety profile.

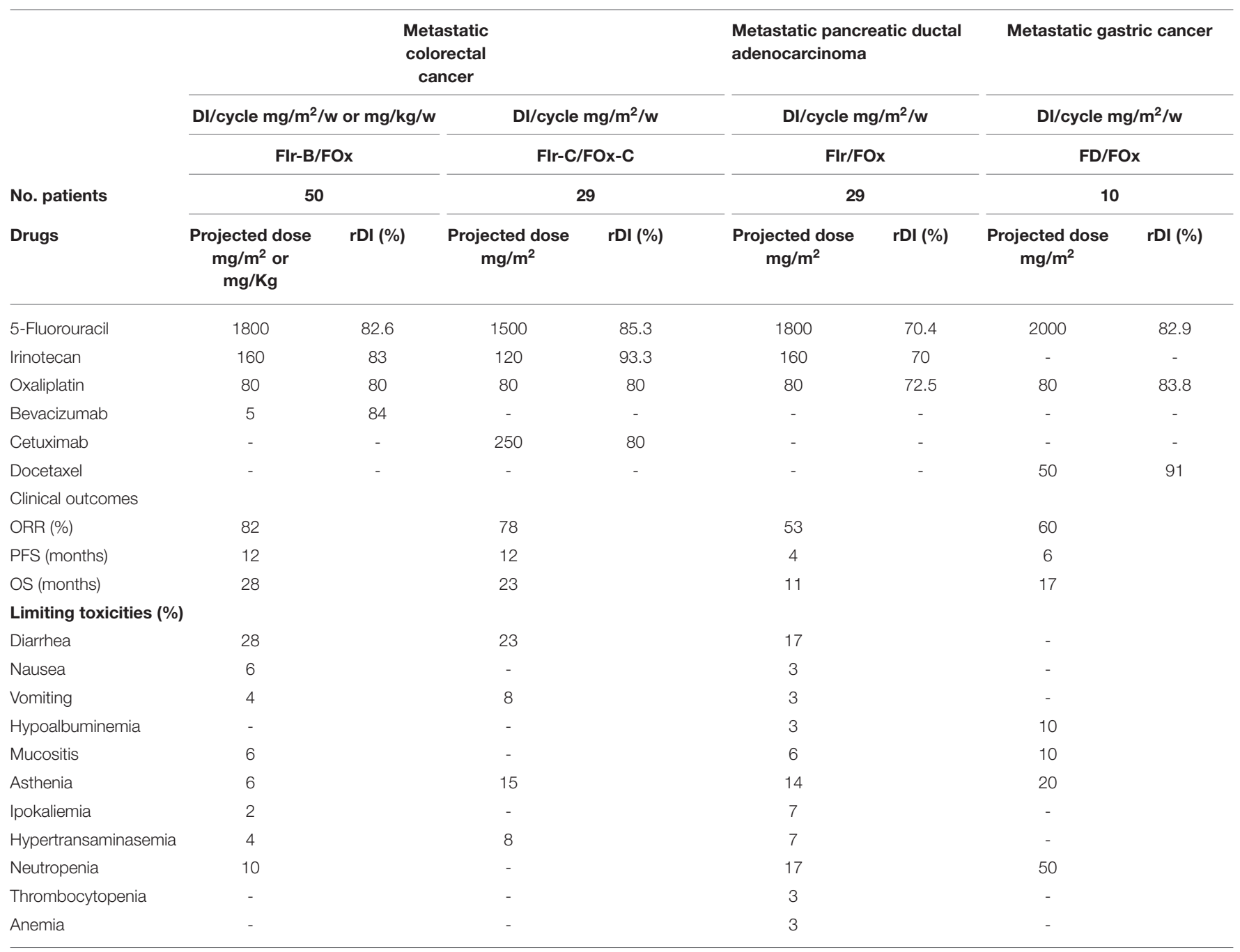

DI, dose-intensity; rDI, received dose-intensity; ORR, objective response rate; PFS, progression-free survival; OS, overall survival.

LTS-ss, 2 (7\%) (20). LTS-ms were characterized by: $\geq 2$ LTs, 2 (7\%), LT associated to G2-3 toxicities, 9 (32\%). In elderly patients compared to non-elderly, LTS were significantly represented by LTS-ms vs. LTS-ss (chi square 3.832, P 0.05). LTS were prevalently characterized by G2-3 diarrhea, 9 patients (69.2\%), 8 LTS-ms and 1 LTS-ss. The 2 LTS-ms with double LT were observed in yE patients. The 10 LTS-ms, defined by LT added to other, at least G2, non-limiting toxicities, were prevalently determined by G2-3 diarrhea (90\%), plus G2-3 nausea/vomiting (70\%). The 10 LTS-ss were prevalently characterized by G3 diarrhea (50\%), G3 asthenia, hypertension, hypertransaminasemy, neutropenia, thrombocytopenia $10 \%$, respectively.

In KRAS/NRAS wild-type mCRC patients treated with FIrC/FOx-C, LTS were observed in 19 patients (65.5\%), 5 out of $6 \mathrm{yE}$ (83\%): LTS-ms 17 (59\%), LTS-ss 2 (7\%) (4). LTS were significantly represented by LTS-ms vs. LTS-ss (chi square 7.703, $P$ 0.006). LTS-ms characterized by $\geq 2$ LT 7 (24\%); LT associated to other toxicities 10 (34\%). LTS were prevalently characterized by G3-4 diarrhea and G3 asthenia associated to other toxicities.
In mPDAC patients treated with FIr/FOx, overall LTS were 8 (27.5\%); 5 out of 13 yE/old-elderly (38.4\%); LTS-ms 7 (24.1\%), LTS-ss 1 (3.4\%) (11). LTS-ms > 2 LTs 1 (3.4\%); LT associated to non-limiting toxicities 6 (20.6\%). LTS were not significantly represented by LTS-ms compared to LTS-ss. In mGC patients treated with FD/FOx, overall LTS were observed in 3 patients (30\%), all LTS-ms: 1 out of $4 \mathrm{yE}(25 \%) ; 2$ out of 6 non-elderly (33.3\%) (14).

Furthermore, the analysis of SNPs represented an exploratory analysis in colorectal cancer patients treated with intensive triplet chemotherapy plus cetuximab, according to FIr-C/FOx$\mathrm{C}$ schedule, to better evaluate the safety profile, particularly gastrointestinal. Patients who reported gastrointestinal LTS, prevalently showed reduced fluorouracil degradation rate (FUDR), Single Nucleotide Polimorphisms (SNP) CYP3A4, UGT1A1, and $>1$ positive pharmacogenomic biomarkers. Specifically, the exploratory analysis of 5-FUDR reduction and $A B C B 1, C Y P 3 A 4, D Y P D, U G T 1 A 1$ SNPs evaluated in 14 KRAS/NRAS wild-type mCRC patients (48.3\%) treated with 
TABLE 2 | Individual Toxicity Syndromes: overall LTS, LTS-ms and LTS-SS, according to triplet chemotherapy-based regimen.

\begin{tabular}{|c|c|c|c|c|c|c|c|c|}
\hline & \multicolumn{4}{|c|}{ Metastatic colorectal cancer } & \multirow{2}{*}{\multicolumn{2}{|c|}{$\begin{array}{c}\text { Metastatic pancreatic ductal } \\
\text { adenocarcinoma }\end{array}$}} & \multirow{2}{*}{\multicolumn{2}{|c|}{$\begin{array}{c}\begin{array}{c}\text { Metastatic gastric } \\
\text { cancer }\end{array} \\
\text { FD/FOx }\end{array}$}} \\
\hline & \multicolumn{2}{|c|}{ FIr-B/FOx } & \multicolumn{2}{|c|}{ FIr-C/FOx-C } & & & & \\
\hline & N. & $\%$ & N. & $\%$ & N. & $\%$ & N. & $\%$ \\
\hline Overall patients & 50 & & 29 & & 29 & & 10 & \\
\hline Toxicity syndromes & 22 & 44 & 19 & 65.5 & 8 & 27.5 & 3 & 30 \\
\hline LTS-ms & 12 & 24 & 17 & 59 & 7 & 24.1 & 3 & 30 \\
\hline LTS-SS & 10 & 20 & 2 & 7 & 1 & 3.4 & - & - \\
\hline Young-elderly patients & 28 & 42 & 6 & 24 & 13 & 34.4 & 4 & 40 \\
\hline Toxicity syndromes & 13 & 46 & 5 & 83 & 5 & 38.4 & 1 & 25 \\
\hline LTS-ms & 11 & 39 & 4 & 67 & 5 & 38.4 & 1 & 25 \\
\hline LTS-SS & 2 & 7 & 1 & 17 & - & - & - & - \\
\hline
\end{tabular}

LTS, limiting toxicity syndromes; LTS-ms, LTS multiple sites; LTS-SS, LTS single site.

FIr-C/FOx-C $(4,24,25)$, and compared with LTS occurrence in $47.4 \%$ of patients who showed LTS, reported prevalent 5FUDR reduction, and CYP3A4, UGT1A1 SNPs in patients with gastrointestinal LTS; 78\% of mCRC patients with LTS showed $>1$ pharmacogenomic alterations, including reduced 5-FUDR, CYP3A4 and/or UGT1A1 SNPs (range 1-3).

\section{DISCUSSION: CLINICAL RELEVANCE OF THE INTEGRATION OF TS IN THE EVALUATION OF TOXICITY}

Over the past 10 years, we developed intensive triplet chemotherapy-based regimens in fit $\mathrm{mGI}$ cancer patients (2, $4,11,14)$, showing activity and clinical outcomes similar to that reported $(3,5-10,12,13,15-18)$, and characterized by FIr/FOx schedule design consisting of weekly administration of 5-FU and weekly alternated administration of two other drugs, such as CPT-11 or OXP (22), in order to recommend schedules providing an adequate balance between received DI $\geq 80 \%$ and treatment-related toxicity (1). Clinical status of the individual patient, also depending from metastatic tumor extension, is the most important variable justifying differential toxicity in individual patients. Thus, patients treated with triplet chemotherapy-based regimens should be enrolled by careful decision-making including age, PS, and comorbidity status (19, 20, 26, 27).

To this aim, FIr-B/FOx (2) as first line treatment of mCRC patients gained equivalent efficacy than that reported with triplet schedules, such as FOLFOXIRI/BEV $(2,3,28)$, and demonstrated a good tolerability profile $(1,29)$, with lower G3-4 neutropenia, also in $\mathrm{yE}$ mCRC patients $(20,27)$. C addiction according to FIr-C/FOx-C schedule in KRAS/NRAS wild-type mCRC (4) met the projected high activity, as different other schedules of $\mathrm{C}$ addition to triplet chemotherapy, chrono-IFLO, ERBIRINOX, FOLFOXIRI (5-8), or panitumumab addiction to modified FOLFOXIRI $(9,10)$, even if individual toxicity profile limited the wide use of intensive schedules associating triplet chemotherapy and anti-EGFR targeted agents in clinical practice, thus requiring pharmacogenomic analysis to more properly select fit mCRC patients (4). FIr/FOx schedule in mPDAC patients may increase activity and efficacy, as previously reported in mCRC patients (11), even if it required modulation of doses reducing median rDI $<80 \%$, and PS 2 may affect significantly worse OS. FD/FOx schedule in mGC patients was feasible at median $\mathrm{rDI}>80 \%$, and showed equivalent efficacy as D associated to cisplatin/5-FU $(16,17)$, with good tolerability (14). In randomized studies, D addiction to cisplatin/5-FU-based triplet chemotherapy regimen $(16,17)$, showed significantly increased toxicity, that limited the expected efficacy of the triplet regimen.

The conventional way to measure toxicity of cancer treatments is represented by the description of each type of toxicity according to National Cancer Institute Common Toxicity Criteria (NCI-CTC, version 4.0), to obtain the description of cumulative and prevalent limiting (G3$\mathrm{G} 4$ ), moderate (G2), mild (G1), or absent toxicities directly determined by the cancer treatment, according to the number of administered cycles and treated patients. This way does not really represent the clinical burden of toxicity in the individual patient, nor define prevalent individual TS affecting a patient population equivalently treated and their variability. Thus, in reported intensive first line triplet chemotherapy-based regimens developed in mGI cancer patients, we added the description of individual TS, and specifically of LTS, to describe cumulative and individual toxicity, that include differential spectrum and intensities of TS, depending from medical treatment and patients' individual clinical conditions.

The integration of the evaluation of LTS to the conventional treatment-related toxicity, contributed a patient-related clinical indicator of toxicity burden, providing a global evaluation of patient-related limiting toxicity $(2,4,11,14,20)$ : in mCRC patients treated with FIr-B/FOx or FIr-C/FOx-C, LTS were 44 and $65.5 \%$, respectively; in mPDAC patients treated with FIr/FOx, 27.5\%; in mGC patients treated with FD/FOx, 30\%. LTS also provided a classification of LTS according to the spectrum and intensity of toxicities: in mCRC patients treated with FIr$\mathrm{B} / \mathrm{FOx}$, LTS-ms were equivalent to LTS-ss; in mCRC patients treated with FIr-C/FOx-C, LTS-ms were significantly prevalent 
$59 \%$ and frequently characterized by LTS with $\geq 2$ LT $24 \%$, and they were also prevalent in mPDAC patients treated with FIr/FOx and in $\mathrm{mGC}$ patients treated with FD/FOx.

In $y E$ patients, LTS evaluation showed the differential tolerability of intensive triplet chemotherapy based regimens: in mCRC, FIr-B/FOx LTS $46 \%$, but significantly prevalent LTSms (20, 27); FIr-C/FOx-C LTS $83 \%$, pointing elderly status $\geq 65$ years as an exclusion criteria for intensive regimens adding triplet chemotherapy to anti-EGFR. In mPDAC patients, FIr/FOx LTS 38.4\%; in mGC patients treated with FD/FOx, LTS $25 \%$. Specifically, LTS also provided an indicator of individual, patient-related toxicity useful for proper treatment and care in clinical practice.

Furthermore, even if performed in only 14 patients $(48.3 \%$ treated) and in 9 who showed LTS (47.4\%), exploratory data of pharmacogenomic biomarkers compared with LTS occurrence in KRAS/NRAS wild-type MCRC patients treated with FIr-C/FOx$\mathrm{C}$ showed that reduced 5-FUDR, and CYP3A4 and UGT1A1 SNPs may predict individual LTS occurrence, particularly at recommended doses, specifically gastrointestinal LTS (4). Thus, LTS can represent the innovative and proper indicator to whom relate pharmacogenomic analysis and it may guide proper selection of patients suitable for intensive regimens adding triplet chemotherapy and anti-EGFR drug, or to modulate intensive triplet chemotherapy-based regimens.

Thus, LTS meets the need of an innovative clinical parameter of patient-related toxicity burden, to measure personalized safety of intensive first line triplet chemotherapy-based regimens proposed in $\mathrm{mGI}$, also associated to targeted agents (B or C). Its clinical relevance should be prospectively evaluated as a model in clinical practice. The integration of LTS and conventional toxicity evaluations may help proper selection of patients fit for intensive first line medical treatments in mGI cancers, to more properly weigh toxicity analysis with activity and clinical outcome and its contribution to address selection of patients suitable for intensive triplet chemotherapy-based regimens. The equivalent, integrated evaluation and monitoring of individual safety by LTS, can help properly select first line intensive medical treatment, and safely administer and manage an intensive first line regimen, that could guarantee increased clinical outcomes and good safety profile in real life.

In the era of precision oncology, integrating molecular characterization of cancer affecting the individual patient to specifically address targeted treatments, such as in mCRC (3032 ), the addition of LTS could integrate the description of cumulative toxicities, toward a precision toxicity evaluation, even to better evaluate innovative drugs as intensive combinations of multiple drugs, favoring the dissemination of innovative treatments in clinical practice.

Analysis of TS could be integrated in the therapeutic pathway of cancer patients in clinical practice and in clinical studies to globally evaluate tolerability of cancer treatments. It could be, also, particularly useful for a more proper evaluation of tolerability in the therapeutic pathway of individual cancer patients unfit for standard treatments, due to elderly status and/or their clinical status, or unfit for intensive regimens (33, 34 ), to optimize simultaneous care of cancer patients (35), and in patients treated with innovative non-infusional targeted-drugs, as well as in adjuvant treatments of early cancers, to even more properly weigh the balance between adjunctive efficacy of cancer treatment and its safety in potentially curable patients.

More, in intensive regimens such as FIr-C/FOx-C, characterized by high discontinuation rate of treatment, highly prevalent (>50\%) LTS and heterogeneity of LT, LTS may help directly relate individual patient- and drugs-related toxicity with pharmacogenomics biomarkers referred to the individual genetic identity of the cancer patient.

\section{CONCLUSION}

TS, specifically LTS, represents an innovative clinical parameter of cumulative and individual patient-related toxicity burden, defining differential spectrum and intensities of treatmentrelated TS, depending from the clinical status of the individual cancer patient, particularly according to elderly, and PS.

\section{DATA AVAILABILITY STATEMENT}

The raw data supporting the conclusions of this article will be made available by the authors, without undue reservation, to any qualified researcher.

\section{ETHICS STATEMENT}

Proposed regimens were approved by Agenzia Italiana del Farmaco for administration in label for treatment in Italian public hospitals, and published in Gazzetta Ufficiale Repubblica Italiana (Elenco dei Medicinali erogabili a totale carico del Servizio Sanitario Nazionale, Gazzetta Ufficiale Repubblica Italiana N.1, 2 Gennaio 2009). MCRC clinical trials were approved by Local Ethical Committee (Comitato Etico, Azienda Sanitaria Locale n.4 L'Aquila, Regione Abruzzo, Italia), and conducted in accordance with Declaration of Helsinki. PDAC study was approved by the Regional Review Board (Regione Abruzzo, Italia, according to D.G.R. n.489, 25/05/2007). All patients provided written, informed consent concerning the proposed treatment, and biological evaluations.

\section{AUTHOR CONTRIBUTIONS}

GB contributed in conceptualization, data curation, formal analysis, investigation, methodology, project administration, validation, and writing original draft. ER contributed in conceptualization, data curation, formal analysis, investigation, methodology, project administration, supervision, validation, and writing-review original draft.

\section{ACKNOWLEDGMENTS}

Preliminary data were previously reported at: 2019 ASCO Meeting, Pubblication-only, J Clin Oncol 37, 2019 (suppl; abstr e15103); 2019 ESMO WGI Congress, Poster Presentation, Annals of Oncology 2019; 30 (Suppl.4), Abstract P-025. 


\section{REFERENCES}

1. Bruera G, Ricevuto E. Intensive chemotherapy of metastatic colorectal cancer: weighing between safety and clinical efficacy. Evaluation of Masi G, Loupakis F, Salvatore L, et al. Bevacizumab with FOLFOXIRI (irinotecan, oxaliplatin, fluorouracil, and folinate) as first-line treatment for metastatic colorectal cancer: a phase 2 trial. Lancet Oncol 2010;11:845-52. Expert Opin. Biol. Ther. (2011) 11:821-4. doi: 10.1517/14712598.2011.582462

2. Bruera G, Santomaggio A, Cannita K, Lanfiuti Baldi P, Tudini M. De Galitiis F, et al. "Poker" association of weekly alternating 5-Fluorouracil, Irinotecan, Bevacizumab and Oxaliplatin (FIr-B/FOx) in first line treatment of metastatic colorectal cancer: a phase II study. BMC Cancer. (2010) 10:567. doi: 10.1186/1471-2407-10-567

3. Loupakis F, Cremolini C, Masi G, Lonardi S, Zagonel V, Salvatore L, et al. Initial therapy with FOLFOXIRI and bevacizumab for Metastatic Colorectal Cancer. N. Engl. J. Med. (2014) 371:1609-18. doi: 10.1056/NEJMoa1403108

4. Bruera G, Massacese S, Pepe F, Malapelle U, Dal Mas A, Ciacco E, et al. Intensive first line FIr-C/FOx-C association of triplet chemotherapy plus cetuximab in RAS wild-type metastatic colorectal cancer patients: preliminary phase II data and prediction of individual limiting toxicity syndromes by pharmacogenomic biomarkers. Ther. Adv. Med. Oncol. (2019) 11:178835919846421. doi: 10.1177/1758835919846421

5. Garufi C, Torsello A, Tumolo S, Ettorre GM, Zeuli M, Campanella C, et al. Cetuximab plus chronomodulated irinotecan, 5-fluorouracil, leucovorin and oxaliplatin as neoadiuvant chemotherapy in colorectal liver metastases: POCHER trial. Br. J. Cancer. (2010) 103:1542-7. doi: 10.1038/sj.bjc.6605940

6. Assenat E, Desseigne F, Thezenas S, Viret F, Mineur L, Kramar A, et al. Cetuximab plus FOLFIRINOX (ERBIRINOX) as first-line treatment for unresectable Metastatic Colorectal Cancer: a phase II trial. Oncologist. (2011) 16:1557-64. doi: 10.1634/theoncologist.2011-0141

7. Saridaki Z, Androulakis N, Vardakis N, Vamvakas L, Kabouraki E, Kalbakis $\mathrm{K}$, et al. A triplet combination with irinotecan (CPT-11), oxaliplatin (LOHP), continuous infusion 5-fluorouracil and leucovorin (FOLFOXIRI) plus cetuximab as first-line treatment in KRAS wt, metastatic colorectal cancer: a pilot phase II trial. Br. J. Cancer. (2012) 107:1932-7. doi: 10.1038/bjc.2012.509

8. Folprecht G, Hamann S, Schütte K, Tratbach T, Stoehlmacher-Williams J, Ehninger G. Dose escalating study of cetuximab and 5-FU/folinic acid (FA)/oxaliplatin/irinotecan (FOLFOXIRI) in first line therapy of patients with metastatic colorectal cancer. BMC Cancer. (2014) 14:521. doi: 10.1186/1471-2407-14-521

9. Fornaro L, Lonardi S, Masi G, Loupakis F, Bergamo F, Salvatore $\mathrm{L}$, et al. FOLFOXIRI in combination with panitumumab as first-line treatment in quadruple wild-type (KRAS, NRAS, HRAS, BRAF) metastatic colorectal cancer patients: a phase II trial by the Gruppo Oncologico Nord Ovest (GONO). Ann. Oncol. (2013) 24:2062-7. doi: 10.1093/annonc/ mdt 165

10. Bendell JC, Zakaru A, Peyton JD, Boccia R, Moskowitz M, Gian V, et al. A Phase II Study of FOLFOXIRI Plus Panitumumab Followed by evaluation for resection in patients with metastatic KRAS wild-type colorectal cancer with liver metastases only. Oncologist. (2016) 21:279-80. doi: 10.1634/theoncologist.2015-0439

11. Bruera G, Massacese S, Candria S, Galvano A, Manetta R, Giordano $\mathrm{AV}$, et al. Real life triplet FIr/FOx chemotherapy in first line metastatic pancreatic ductal adenocarcinoma patients: recommended schedule for expected activity and safety and phase II study. Oncotarget. (2018) 9:31861-76. doi: 10.18632/oncotarget. 25870

12. Von Hoff DD, Ervin T, Arena FP, Chiorean EG, Infante J, Morre M, et al. Increased survival in pancreatic cancer with nab-paclitaxel plus gemcitabine. N. Engl. J. Med. (2013) 369:1691-703. doi: 10.1056/NEJMoa1 304369

13. Conroy T, Desseigne F, Ychou M, Bouche O, Guimbaud R, Becouam Y, et al. FOLFIRINOX versus gemcitabine for metastatic pancreatic cancer. N. Engl. J. Med. (2011) 364:1817-25. doi: 10.1056/NEJMoa1011923

14. Bruera G, Massacese S, Galvano A, Dal Mas A, Guadagni S, Ciacco E, et al. Dose-finding study of intensive weekly alternating schedule of docetaxel, 5fluorouracil, and oxaliplatin, FD/FOx regimen, in metastatic gastric cancer. Oncotarget. (2018) 9:20339-50. doi: 10.18632/oncotarget.24861
15. Al-Batran SE, Hartmann JT, Probst S, Schmalenberg H, Hollerbach $\mathrm{S}$, Hofheinz R, et al. Phase III trial in metastatic gastroesophageal adenocarcinoma with fluorouracil, leucovorin plus either oxaliplatin or cisplatin: a study of the Arbeitsgemeinschaft Internistische Onkologie. J. Clin. Oncol. (2008) 26:1435-42. doi: 10.1200/JCO.2007.13.9378

16. Van Cutsem E, Moiseyenko VM, Tjulandin S, Majlis A, Constenla M, Boni C, et al. Phase III study of docetaxel and cisplatin plus fluorouracil compared with cisplatin and fluorouracil as first-line therapy for advanced gastric cancer: a report of the V325 Study Group. J. Clin. Oncol. (2006) 24:4991-7. doi: 10.1200/JCO.2006.06.8429

17. Al-Batran SE, Hartmann JT, Hofheinz R, Homann N, Rethwisch V, Probst S, et al. Biweekly fluorouracil, leucovorin, oxaliplatin, and docetaxel (FLOT) for patients with metastatic adenocarcinoma of the stomach or esophagogastric junction: a phase II trial of the Arbeitsgemeinschaft Internistische Onkologie. Ann. Oncol. (2008) 19:1882-7. doi: 10.1093/annonc/mdn403

18. Bang YJ, Van Cutsem E, Feyereislova A, Chung HC, Shen L, Sawaki A, et al. Trastuzumab in combination with chemotherapy versus chemotherapy alone for treatment of HER2-positive advanced gastric or gastro-oesophageal junction cancer (ToGA): a phase 3, open-label, randomised controlled trial. Lancet. (2010) 376:687-97. doi: 10.1016/S0140-6736(10)61121-X

19. Extermann M, Overcash J, Lyman GH, Parr J, Balducci L. Comorbidity and functional status are independent in older cancer patients. J. Clin. Oncol. (1998) 16:1582-7. doi: 10.1200/JCO.1998.16.4.1582

20. Bruera G, Cannita K, Giordano AV, Vicentini R, Ficorella R, Ricevuto E. Effectiveness and safety of intensive triplet chemotherapy plus bevacizumab, FIr-B/FOx, in young-elderly Metastatic Colorectal Cancer (MCRC) patients. BioMed. Res. Int. (2013) 2013:143273. doi: 10.1155/2013/143273

21. Ficorella C, Ricevuto E, Morelli MF, Morese R, Cannita K, Cianci G, et al. Increased tolerability of bimonthly $12 \mathrm{~h}$ timed flat infusion 5fluorouracil/irinotecan regimen in advanced colorectal cancer: a dose finding study. Oncol. Rep. (2006) 15:1345-50. doi: 10.3892/or.15.5.1345

22. Morelli MF, Santomaggio A, Ricevuto E, Cannita K, De Galitiis F, Tudini M, et al. Triplet schedule of weekly 5-Fluorouracil and alternating irinotecan or oxaliplatin in Advanced Colorectal Cancer: a dose-finding and phase II study. Oncol. Rep. (2010) 23:1635-40. doi: 10.3892/or_00000805

23. Mantel N. Chi-square tests with one degree of freedom: extensions of the Mendel-Haenszel procedure. J. Am. Statist. Assoc. (1963) 58:690-700. doi: 10.1080/01621459.1963.10500879

24. Falvella FS, Cheli S, Martinetti A, Mazzali C, Iacovelli R, Maggi C, et al. DPD and UGT1A1 deficiency in colorectal cancer patients receiving triplet chemotherapy with fluoropyrimidines, oxaliplatin, and irinotecan. Br. J. Clin. Pharmacol. (2015) 80:581-8. doi: 10.1111/bcp.12631

25. Dias MM, McKinnon RA, Sorich MJ. Impact of the UGT1A1*28 allele on response to irinotecan: a systematic review and meta-analysis. Pharmacogenomics. (2012) 13:889-99. doi: 10.2217/pgs.12.68

26. Bruera G, Cannita K, Di Giacomo D, Lamy A, Troncone G, Dal Mas A, et al. Prognostic value of KRAS genotype in metastatic colorectal cancer (MCRC) patients treated with intensive triplet chemotherapy plus bevacizumab (FIrB/FOx) according to extension of metastatic disease. BMC Med. (2012) 10:135. doi: 10.1186/1741-7015-10-135

27. Bruera G, Russo A, Galvano A, Rizzo S, Ricevuto E. Clinical parameters to guide decision-making in elderly metastatic colorectal cancer patients treated with intensive cytotoxic and anti-angiogenic therapy. Oncotarget. (2017) 8:37875-83. doi: 10.18632/oncotarget.14333

28. Bruera G, Cannita K, Giuliante F, Lanfiuti Baldi P, Vicentini R, Marchetti $\mathrm{P}$, et al. Effectiveness of liver metastasectomies in Metastatic Colorectal Cancer (MCRC) patients treated with triplet chemotherapy plus bevacizumab (FIr-B/FOx). Clin. Colorectal Cancer. (2012) 11:119-26. doi: 10.1016/j.clcc.2011.11.002

29. Ficorella C, Bruera G, Cannita K, Porzio G, Lanfiuti Baldi P, Tinari N, et al. Triplet Chemotherapy in patients with metastatic colorectal cancer: toward the best way to safely administer a highly active regimen in clinical practice. Clin. Colorectal Cancer. (2012) 11:229-37. doi: 10.1016/j.clcc.2012.05.001

30. Bruera G, Cannita K, Di Giacomo D, Lamy A, Frebourg T, Sabourin JC, et al. Worse prognosis of KRAS c. $35 \mathrm{G}>\mathrm{A}$ mutant metastatic colorectal cancer (MCRC) patients treated with intensive triplet chemotherapy plus bevacizumab (FIr-B/FOx). BMC Med. (2013) 11:59. doi: 10.1186/1741-7015-11-59 
31. Bruera G, Cannita K, Tessitore A, Russo A, Alesse E, Ficorella C, et al. The prevalent KRAS exon 2 c. $35 \mathrm{G}>\mathrm{A}$ mutation in metastatic colorectal cancer patients: a biomarker of worse prognosis and potential benefit of bevacizumab-containing intensive regimens? Crit. Rev. Oncol. Hematol. (2015) 93:190-202. doi: 10.1016/j.critrevonc.2014. 10.004

32. Bruera G, Pepe F, Malapelle U, Pisapia P, Dal Mas A, Di Giacomo D, et al. KRAS, NRAS, and BRAF mutations detected by next generation sequencing, and differential clinical outcome in metastatic colorectal cancer (MCRC) patients treated with first line FIr-B/FOx adding bevacizumab (BEV) to triplet chemotherapy. Oncotarget. (2018) 9:26279-90. doi: 10.18632/oncotarget. 25180

33. Bruera G, Cannita K, Giordano AV, Vicentini R, Ficorella C, Ricevuto E. Differential prognosis of metastatic colorectal cancer patients post-progression to first line triplet chemotherapy plus bevacizumab, FIr-B/FOx, according to second line treatment and KRAS genotype. Int. J. Oncol. (2014) 44:17-26. doi: 10.3892/ijo.2013. 2179
34. Bruera G, Cannita K, Giordano AV, Vicentini R, Ficorella C, Ricevuto E. Prognostic relevance of KRAS genotype in metastatic colorectal cancer patients unfit for FIr-B/FOx intensive regimen. Int. J. Oncol. (2014) 44:182030. doi: $10.3892 /$ ijo.2014.2369

35. Numico G, Cristofano A, Mozzicafreddo A, Cursio OE, Franco P, Courthod $\mathrm{G}$, et al. Hospital admission of cancer patients: avoidable practice or necessary care? PLoS ONE. (2015) 10:e0120827. doi: 10.1371/journal.pone.0120827

Conflict of Interest: The authors declare that the research was conducted in the absence of any commercial or financial relationships that could be construed as a potential conflict of interest.

Copyright (c) 2020 Bruera and Ricevuto. This is an open-access article distributed under the terms of the Creative Commons Attribution License (CC BY). The use, distribution or reproduction in other forums is permitted, provided the original author(s) and the copyright owner(s) are credited and that the original publication in this journal is cited, in accordance with accepted academic practice. No use, distribution or reproduction is permitted which does not comply with these terms. 